\title{
THE DISTRIBUTION OF RATIONAL NUMBERS ON CANTOR'S MIDDLE THIRDS SET
}

\author{
Alexander D. Rahm ${ }^{1}$ - Noam Solomon ${ }^{2}$ - Tara Trauthwein ${ }^{3}$ - \\ BARAK WEISS ${ }^{4}$ \\ ${ }^{1}$ University of French Polynesia, GAATI Laboratory, Faa'a, FRENCH POLYNESIA \\ ${ }^{2}$ Massachusetts Institute of Technology, Cambridge, MA, U.S.A \\ ${ }^{3}$ University of Luxembourg, Department of Mathematics, Esch-sur-Alzette, LUXEMBOURG \\ ${ }^{4}$ Tel Aviv University, Department of Mathematics, Tel-Aviv, ISRAEL
}

\begin{abstract}
We give a heuristic argument predicting that the number $N^{*}(T)$ of rationals $p / q$ on Cantor's middle thirds set $\mathcal{C}$ such that $\operatorname{gcd}(p, q)=1$ and $q \leq T$, has asymptotic growth $O\left(T^{d+\varepsilon}\right)$, for $d=\operatorname{dim} \mathcal{C}$. Our heuristic is related to similar heuristics and conjectures proposed by Fishman and Simmons. We also describe extensive numerical computations supporting this heuristic. Our heuristic predicts a similar asymptotic if $\mathcal{C}$ is replaced with any similar fractal with a description in terms of missing digits in a base expansion. Interest in the growth of $N^{*}(T)$ is motivated by a problem of Mahler on intrinsic Diophantine approximation on $\mathcal{C}$.
\end{abstract}

\section{Communicated by Yann Bugeaud}

\section{Introduction}

Let $\mathcal{C}$ denote Cantor's middle thirds set, i.e., all numbers represented as

$$
x=\sum_{1}^{\infty} a_{i} 3^{-i} \quad \text { with } \quad a_{i}=a_{i}(x) \in\{0,2\} \text { for all } i .
$$

(C) 2020 BOKU-University of Natural Resources and Life Sciences and Mathematical Institute, Slovak Academy of Sciences.

2010 Mathematics Subject Classification: 11K60: Diophantine approximation in probabilistic number theory.

Keywords: Rational numbers in the Cantor set.

A. D. Rahm would like to thank Steve Gelbart and the Weizmann Institute of Science for funding the initial part, as well as Gabor Wiese and the University of Luxembourg for funding the final part of his contribution to this research project. The research of B. Weiss was supported by ISF grant 2095/15 and BSF grant 2016256 .

Licensed under the Creative Commons Attribution-NC-ND 4.0 International Public License. 
Let $N^{*}(T)$ denote the number of rationals number of the form $p / q$, with $p$ and $q$ coprime, which belong to $\mathcal{C}$ and for which $0<q \leq T$. Motivated by questions in Diophantine approximation, our goal will be to understand the asymptotic growth rate of $N^{*}(T)$.

Everything we will say in the sequel will apply with minor modifications to a more general situation in which $\mathcal{C}$ is the set of numbers defined by a restriction in a digital expansion, i.e., for some integer $b \geq 3$ and some proper subset $\mathcal{F}$ of $\{0, \ldots, b-1\}$ we will let $\mathcal{C}$ denote the set of numbers $x=\sum_{1}^{\infty} a_{i} b^{-i}$ with all $a_{i} \in \mathcal{F}$. To simplify notation we will stick throughout to the standard ternary set. When writing a rational as $p / q$ we always assume that $p$ and $q$ are coprime.

Fix $c \in(0,1)$, let $I_{T}$ denote the interval $[(1-c) T, T]$ and let

$$
\begin{aligned}
& N(T) \stackrel{\text { def }}{=} \#\left\{\frac{p}{q} \in \mathcal{C}: q \in I_{T}\right\}, \\
& \widetilde{N}(T) \stackrel{\text { def }}{=} \#\left\{\frac{p}{q} \in \mathcal{C} \text { purely periodic }: q \in I_{T}\right\}, \\
& \widetilde{N}^{*}(T) \stackrel{\text { def }}{=} \#\left\{\frac{p}{q} \in \mathcal{C}: 0<q \leq T, \frac{p}{q} \text { is purely periodic }\right\} .
\end{aligned}
$$

Note that these quantities depend on $c$ but this will be suppressed from the notation. The notations $A(T)=O(B(T))$ and $A(T) \ll B(T)$ mean that $A(T) / B(T)$ is bounded above by a positive constant, and $A(T) \asymp B(T)$ means that the $A(T) \ll B(T) \ll A(T)$.

ConjeCture 1. Let $d$ be the Hausdorff dimension of $\mathcal{C}$, i.e., $d=\log 2 / \log 3$, and in the general case, $d=\log |\mathcal{F}| / \log b$. For each $\varepsilon>0$ we have $\widetilde{N}(T)=O\left(T^{d+\varepsilon}\right)$.

This conjecture was also made by Broderick, Fishman and Reich in [BFR], and futher heuristics and conjectures which imply Conjecture 1 were given by Fishman and Simmons in [FS, §5]. An upper bound $N(T)=O\left(T^{2 d}\right)$ was obtained by Schleischitz in [Sch, Thm. 4.1].

Our heuristic actually predicts a more precise upper bound for $\widetilde{N}(T)$, see Remark 4.1. The exponent $d$ is optimal in view of Proposition 3.3.

Since numbers in $\mathcal{C}$ are explicitly given in terms of their base 3 expansion, it is possible to count their number as a function of the complexity of their base 3 expansions. But this says nothing about the denominator $q$ in reduced form; it may happen that a rational with a complicated base 3 expansion corresponds to a reduced fraction $p / q$ with $q$ small. The basic heuristic principle behind Conjecture1, is that the two events of having a small denominator relative to the complexity of the base 3 expansion, and of belonging to $\mathcal{C}$, are probabilistically independent. We will make this heuristic more precise below. 
Some computational evidence for Conjecture 1 is given in BFR. Our goal in this paper is to present more evidence supporting it. We will prove that the conjectured asymptotics are lower bounds for $N^{*}(T)$ and $\widetilde{N}^{*}(T)$; we will describe extensive computations consistent with this conjecture; and we will discuss the heuristic motivating Conjecture 1, exhibiting some numerical results which lend some support to this heuristic.

\section{Organization of the paper}

In $\S 2$ we discuss some problems in Diophantine approximation which led us to this problem, and derive a Diophantine consequence from Conjecture 1 . In $\S 3$ we discuss basic properties of base 3 expansions, which yield lower bounds on $N^{*}(T)$ and $\widetilde{N}^{*}(T)$. We also explain that the main quantity of interest is $\widetilde{N}(T)$. In $\S$ 田 we introduce a simple probabilistic model and use it to predict $\widetilde{N}(T)$. Some oversimplifications in the probabilistic models lead to incorrect predictions, and we modify the model slightly in $\S 5$ to remedy this, at the same time showing that the revised model makes the same predictions for the growth of the expectation of $\widetilde{N}(T)$. We discuss fluctuations and the relation of expectations to asymptotic behavior, in $\S 6$. Our computational evidence for our conjectures are given throughout the paper.

\section{Motivation and historical background}

The classical problem in Diophantine approximation may be formulated as follows. Given a decreasing function $\varphi: \mathbb{R}_{+} \rightarrow \mathbb{R}_{+}$and a real number $x$, are there infinitely many rationals $p / q$ such that $|x-p / q|<\varphi(q)$ ? In case this holds one says that $x$ is $\varphi$-approximable. For some choices of $x$ and $\varphi$, determining whether $x$ is $\varphi$-approximable is considered hopelessly difficult (e.g. $\varphi(q)=10^{-100} / q^{2}$, with $x=2^{1 / 3}$ or $\pi$ ); a fruitful line of research is to fix $\varphi$ and ask about the measure of $\varphi$-approximable numbers, with respect to some measure. Some classical results in diophantine approximation are:

(Dirichlet): Every $x$ is $1 / q^{2}$-approximable.

(Khinchin): With respect to Lebesgue measure, if $\sum q \varphi(q)$ converges, then almost no $x$ is $\varphi$-approximable, and if $\sum q \varphi(q)$ diverges, then almost every $x$ is $\varphi$-approximable.

(Jarník): The set

$$
\mathrm{BA} \stackrel{\text { def }}{=}\left\{x: \exists c>0 \text { s.t. } x \text { is not } c / q^{2} \text {-approximable }\right\}
$$

has Hausdorff dimension 1, but Lebesge measure zero. 
One measure to consider in place of Lebesgue measure in such statements, is the coin tossing measure (assigning equal probability $1 / 2$ to the digits 0,2 in base 3 expansion) on Cantor's ternary set $\mathcal{C}$. We give a brief list of activity concerning this type of question.

In 1984, Mahler [M] asked how well numbers in $\mathcal{C}$ can be approximated

(i) by rationals in $\mathbb{R} \backslash \mathcal{C}$.

(ii) by rationals in $\mathcal{C}$.

Question (i) can be formalized in various ways, e.g., for which functions $\varphi$, does $\mathcal{C}$ contain $\varphi$-approximable numbers? For which $\varphi$ is almost every number in $\mathcal{C}$ (with respect to the natural coin-tossing measure) $\varphi$-approximable? For which $\varphi$ is the set of numbers in $\mathcal{C}$ which are $\varphi$-approximable of the same Hausdorff dimension as that of $\mathcal{C}$ ? There has been a lot of recent activity concerning these and similar questions, see [W, $\mathrm{F}, \mathrm{LSV}, \mathrm{Bu},[\mathrm{S}, \mathrm{SW}]$ and the references therein.

Question (ii), which is referred to as an intrinsic approximation problem, has not been nearly as well studied. Broderick, Fishman and Reich BFR proved an analogue of Dirichlet's theorem for Cantor sets and other missing digit sets. Fishman and Simmons [FS extended the main result of BFR to a more general class of fractal subsets of $\mathbb{R}$. A major difficulty in intrinsic approximation problems is that there is no reasonable understanding of the growth of the function $N(T), \widetilde{N}(T)$ as described above; bounds on these functions will yield some progress on Mahler's question (ii).

In particular, Conjecture 1 implies (see [BFR] for the derivation):

ConjeCture 2. For almost every $x \in \mathcal{C}$, with respect to the coin-tossing measure, for any $\varepsilon>0$, there are only finitely many rationals $p / q \in \mathcal{C}$ such that

$$
\left|x-\frac{p}{q}\right|<\frac{1}{q^{1+\varepsilon}} .
$$

It was shown in $\mathrm{BFR}$ that for each $x \in \mathcal{C}$, there are infinitely many $p / q \in \mathcal{C}$ for which $|x-p / q|<q^{-1}(\log q)^{-1 / d}$. Thus the exponent in (2.1) cannot be improved.

\section{Notation, basic observations, and a lower bound}

The number $x=\sum_{1}^{\infty} a_{i}(x) 3^{-i}$ is rational if and only if the sequence $\left(a_{i}(x)\right)_{i \geq 1}$ is eventually periodic, i.e., there are integers $i_{0}=i_{0}(x) \geq 0$ and $\ell=\ell(x)>0$, called, respectively, the length of initial block and period, such that

$$
a_{i}(x)=a_{i+\ell}(x), \text { for all } i>i_{0},
$$

and (3.1) does not hold for any smaller $i_{0}$ or $\ell$. 
We say that $x$ is purely periodic if $i_{0}=0$. It is elementary to verify the following (see also [BFR, Lemma 2.3])

Proposition 3.1. Suppose $x$ is a rational in $\mathcal{C}$, with $\left(a_{i}\right), i_{0}$ and $\ell$ as above. Then we may write $x=P / Q$, where

$$
P=\sum_{j=0}^{i_{0}} a_{j} 3^{i_{0}+\ell-j}-\sum_{j=0}^{i_{0}} a_{j} 3^{i_{0}-j}+\sum_{j=1}^{\ell} a_{i_{0}+j} 3^{\ell-j}, \quad \text { and } Q=3^{i_{0}}\left(3^{\ell}-1\right)
$$

(this fraction need not be reduced). In particular:

- If $x$ is a rational in $\mathcal{C}$ with period $\ell$ and initial block of length $i_{0}$, then there is an integer $N$ such that $3^{i_{0}} x-N$ is a purely periodic rational in $\mathcal{C}$ with period $\ell$.

- if $x=p / q$ is purely periodic, where $\operatorname{gcd}(p, q)=1$, then $q$ is a divisor of $3^{\ell}-1$ and $\ell$ is the order of 3 in the multiplicative group $(\mathbb{Z} / q \mathbb{Z})^{\times}$.

As mentioned above, throughout this paper, the notation $x=p / q$ will mean that $x$ is a reduced rational in $\mathcal{C}$, i.e., $\operatorname{gcd}(p, q)=1$. The notation $x=P / Q$ will mean that $x$ is a rational in $\mathcal{C}$, not necessarily reduced.

The following proposition follows from standard calculations and is left to the reader.

Proposition 3.2. Fix $c, c^{\prime} \in(0,1)$ and define $\tilde{N}(T)$ and $\widetilde{N}^{\prime}(T)$ using $c$ and $c^{\prime}$, respectively. Fix $\varepsilon>0$. If $\tilde{N}(T) \ll T^{d+\varepsilon}$, then the same holds for $\widetilde{N}^{\prime}(T), N(T)$, $\widetilde{N}^{*}(T)$, and $N^{*}(T)$.

Proposition 3.3. There is $c_{1}>0$ such that for all $T>3$ we have $\tilde{N}^{*}(T) \geq$ $T^{d} / 2$ and $N^{*}(T) \geq c_{1} \log (T) T^{d}$.

Proof. Let $\ell=\left\lfloor\log _{3} T\right\rfloor \geq 1$, i.e., $T \in\left[3^{\ell}, 3^{\ell+1}\right]$. There are $2^{\ell}$ purely periodic Cantor rationals of the form $P / Q$ with $Q=3^{\ell}-1$. Bringing them to reduced form, they are of the form $p / q$ with $q \leq T$. In particular

$$
\tilde{N}^{*}(T) \geq 2^{\ell}=\left(3^{\ell+1}\right)^{d} / 2 \geq T^{d} / 2 .
$$

Similarly, any rational of the form $P / Q$, where $Q=3^{i_{0}}\left(3^{\ell-i_{0}}-1\right)$ will contribute to $N^{*}(T)$. For each such $Q$, there are $2^{\ell-i_{0}}$ possibilities for the digits in the periodic part of $P / Q$, and $2^{i_{0}}$ for the digits in the initial block. An exercise involving the inclusion/exclusion principle (which we omit), implies that the repetition in this counting is negligible, i.e., up to a constant, the number of distinct rationals $P / Q$ written in this form is at least $\ell 2^{\ell}$. This proves the claim. 


\section{ALEXANDER D. RAHM-NOAM SOLOMON-TARA TRAUTHWEIN-BARAK WEISS}

\section{The heuristic}

In this section we justify an upper bound of the form $\tilde{N}(T)=O\left(T^{d+\varepsilon}\right)$. Our approach is to assign to each reduced rational $p / q$ a probability that it belongs to $\mathcal{C}$, and bound the expectation of the random variable $\widetilde{N}(T)$ with respect to this probability. For a related approach, and other statements implied by related arguments, see [FS, §5]. Let $Q=3^{\ell}-1$ and consider the rationals $P / Q$ in the interval $[0,1]$. There are $3^{\ell}$ such rationals, and of these, $2^{\ell}$ belong to $\mathcal{C}$. By Proposition 3.1, they are precisely the purely periodic Cantor rationals with period dividing $\ell$. That is, fixing $Q$, the proportion of rationals $P / Q \in[0,1]$ which belong to $\mathcal{C}$ is $\left(\frac{2}{3}\right)^{\ell}$.

Motivated by this we define our probabilistic model. By Proposition 3.1 . $p / q \in \mathcal{C}$ is purely periodic if and only if 3 does not divide $q$. For each rational $p / q \in[0,1]$, with $q$ not divisible by 3 , our model stipulates:

$\left(^{*}\right)$ The probability that $p / q \in \mathcal{C}$ is $\left(\frac{2}{3}\right)^{\ell}$, where $\ell=\ell(q)$ is the smallest number for which $Q=3^{\ell}-1$ is divisible by $q$; the events $p / q \in \mathcal{C}$ are completely independent.

Note that $\ell$ is the order of 3 in the multiplicative group $C_{q} \stackrel{\text { def }}{=}(\mathbb{Z} / q \mathbb{Z})^{\times}$. Let $\phi(q)=\# C_{q}$ be the Euler number of $q$. We may take representatives of elements of $C_{q}$ to be the integers $p$ between 0 and $q-1$ coprime to $q$, so we find that the expected number of $p / q$ in $\mathcal{C}$ with fixed denominator $q$ is $\phi(q)\left(\frac{2}{3}\right)^{\ell(q)}$. Thus

$$
\begin{aligned}
\mathbb{E}(\widetilde{N}(T)) & =\sum_{q \in I_{T}} \phi(q)\left(\frac{2}{3}\right)^{\ell(q)} \\
& \leq \sum_{q \in I_{T}} T\left(\frac{2}{3}\right)^{\ell(q)} \\
& =T \sum_{\ell \geq \log _{3} T+c^{\prime}} \# L(\ell, T)\left(\frac{2}{3}\right)^{\ell},
\end{aligned}
$$

where

$$
L(\ell, T)=\left\{q \in I_{T}: \ell(q)=\ell\right\} \quad \text { and } \quad c^{\prime}=\log _{3}(1-c) .
$$

We now need to bound the terms \#L( $\ell, T)$. First we choose $\lambda=\frac{2-d}{1-d}$. For $\ell \geq \lambda \log _{3} T$ we can use the trivial bound $\# L(\ell, T) \leq T$, since

$$
T^{2} \sum_{\ell \geq \lambda \log _{3} T}\left(\frac{2}{3}\right)^{\ell} \asymp T^{2-\lambda+\lambda d}=T^{d} .
$$


So it only remains to show

$$
T \sum_{\ell=\log _{3} T+c^{\prime}}^{\lambda \log _{3} T} \# L(\ell, T)\left(\frac{2}{3}\right)^{\ell}=O\left(T^{d+\varepsilon}\right) .
$$

For $\ell \in\left[\log _{3} T+c^{\prime}, \lambda \log _{3} T\right]$, we use the obvious inequality

$\# L(\ell, T) \leq \tau\left(3^{\ell}-1\right)$, where $\tau(n)$ denotes the number of divisors of $n$. It is well-known that

$$
\tau(n) \leq 2^{(1+o(1)) \log n / \log \log n} .
$$

In our situation we have $3^{\ell}-1 \leq T^{\lambda}$, so

implying

$$
\tau\left(3^{\ell}-1\right) \leq 2^{2 \lambda \log T / \log \log T}=T^{2 \lambda / \log \log T},
$$

$$
\begin{aligned}
T \sum_{\ell=\log _{3} T+c^{\prime}}^{\lambda \log _{3} T} \# L(\ell, T)\left(\frac{2}{3}\right)^{\ell} & \leq T \lambda \log _{3} T T^{2 \lambda / \log \log T}\left(\frac{2}{3}\right)^{\log _{3} T+c^{\prime}} \\
& \ll \log T T^{d+2 \lambda / \log \log T} .
\end{aligned}
$$

from which (4.2) follows.

\section{REMARK 4.1.}

1. In (4.1) we used the inequality $\phi(q) \leq q \leq T$. But in fact it is well-known that on average $\phi(q) \asymp q$, so we actually expect

$$
\tilde{N}(T) \asymp T \sum_{\ell=\log _{3} T+c^{\prime}}^{\lambda \log _{3} T} \# L(\ell, T)\left(\frac{2}{3}\right)^{\ell} .
$$

2. Our arguments show that the right hand side of (4.4) behaves like

$$
O\left(\log T T^{d+2 \lambda / \log \log T}\right) .
$$

In estimating the cardinality of $L(\ell, T)$ we used the bound (4.3) which is optimal for a general $n$. However it may be that for numbers of the form $n=3^{\ell}-1$ a better bound exists, see $[\mathrm{E}$ for related results. If so, then our heuristic would predict a better bound for $\widetilde{N}(T)$.

\section{A revised model}

The heuristic above relied on the basic statement $\left(^{*}\right)$. However, this assumption leads to some clearly incorrect predictions, namely: 
(i) Primitive words: In deriving $\left(^{*}\right)$ we calculated the frequency of purely periodic rationals with period dividing $\ell$, belonging to $\mathcal{C}$. It would have been more precise to count the purely periodic rationals with period $e x$ actly $\ell$, belonging to $\mathcal{C}$. By Proposition 3.1. rationals with period exactly $\ell$ correspond to primitive words $w$ in the alphabet $\{0,1,2\}$ of length $\ell$, i.e., those $w$ for which there is no proper divisor $k$ of $\ell$ such that $w$ is a concatenation of identical words of length $k$. A standard application of the inclusion/exclusion principle gives that the number of primitive words of length $\ell$ from an alphabet of size $a$ is

$$
m(\ell, a) \stackrel{\text { def }}{=} \sum_{d \mid \ell} \mu\left(\frac{\ell}{d}\right) a^{d},
$$

where $\mu$ is the Möbius function.

(ii) Multiples of $\ell$ : Fix $q$ and let

$$
N_{q}=\#\{p: p / q \in \mathcal{C}\}
$$

and let $\ell=\ell(q)$. Since $\mathcal{C}$ is invariant under multiplication by $3 \bmod 1$, whenever $p / q \in \mathcal{C}$ we also have $p^{\prime} / q \in \mathcal{C}$, where $p^{\prime}=3 p \bmod q$. This means that the set $\{p: p / q \in \mathcal{C}\}$ consists of orbits for the action of 3 on $C_{q}$, and in particular, $\ell$ divides $N_{q}$.

(iii) Divisibility by 2: Let $Q=3^{\ell}-1$. Our model predicts that there are $\phi(Q)(2 / 3)^{\ell}$ rationals in $\mathcal{C}$ with denominator $Q$, coming from $P \in\{0, \ldots$ $\ldots, Q-1\}$ such that $P / Q$ belongs to $\mathcal{C}$ and $\operatorname{gcd}(P, Q)=1$. However $Q$ is even and if $P / Q$ is in $\mathcal{C}$, then so is $P$, since it may be written in base 3 using the letters 0 and 2 only. That is, the actual number is zero. A similar observation holds for any $q$, which divides $Q=3^{\ell}-1$ but does not divide $Q / 2$.

One may define a revised model as follows: for each $q$, let $H$ be the group generated by 3 in $C_{q}$. By observation (ii), for each coset $X \in C_{q} / H$, all number of the form $p / q, p \in X$ simultaneously belong or do not belong to $\mathcal{C}$; if they all do, we will write $X \in \mathcal{C}$. With this notation, our revised model stipulates that:

$\left.{ }^{* *}\right)$ Suppose $q$ is not divisible by 3 and divides $\left(3^{\ell}-1\right) / 2$, where $\ell=\ell(q)$. For each $X \in C_{q} / H$, the probability that $X \in \mathcal{C}$ is $\frac{m(\ell, 2)}{\bar{m}(\ell, 3)}$, where $m(\ell, a)$ is defined by (5.1) and $\bar{m}(\ell, a)$ is the set of primitive words of length $\ell$ in the symbols $\{0,1,2\}$ defining even numbers. 


\section{RATIONALS IN THE CANTOR SET}

Note that our choice of probability takes into account (i) and (iii). It is not hard to show that

$$
\frac{m(\ell, 2) / \bar{m}(\ell, 3)}{(2 / 3)^{\ell}} \rightarrow_{\ell \rightarrow \infty} 2,
$$

and using this, that the arguments given in $\S 4$ also apply to the second model, yielding the same prediction. That is, model $\left({ }^{* *}\right)$ also implies Conjecture 1 . Moreover, when $\ell$ is prime, it is easy to check using (5.1) and the definition of $\bar{m}$ that the difference between $2\left(\frac{2}{3}\right)^{\ell}$ and $\frac{m(\ell, 2)}{\bar{m}(\ell, 3)}$ is negligible. Nevertheless, when testing our heuristic, there will be a difference between models $\left(^{*}\right)$ and $\left(^{* *}\right)$. For sufficiently small values of $q$ we have computed the actual values of $N_{q}$ as defined in (5.2), and one may compare them to the number

$$
\operatorname{MLO}(q) \stackrel{\text { def }}{=} \operatorname{round}\left(\frac{\phi(q) \cdot m(\ell, 2)}{\bar{m}(\ell, 3)}\right) .
$$

See Figures 1 and 2 ,

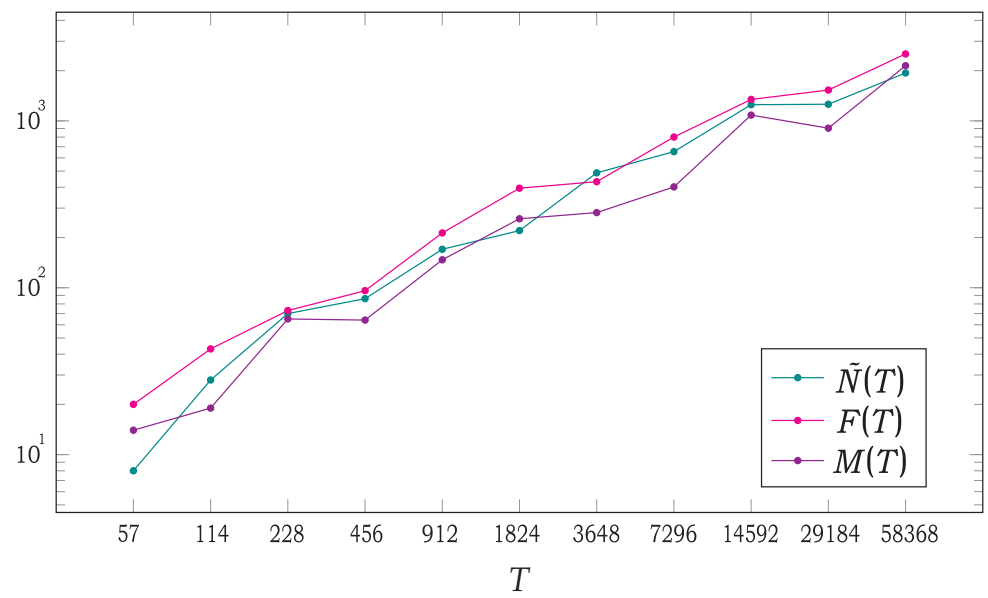

FiguRE 1. The summed number of purely periodic Cantor rationals $\tilde{N}(T)$, its approximation $F(T):=\sum_{\substack{q \in I_{T} \\ 3 \nmid q}} \operatorname{round}\left(\left(\frac{2}{3}\right)^{\ell(q)} \cdot 2 \cdot \phi(q)\right)$ from model $\left({ }^{*}\right)$, and its approximation $M(T):=\sum_{q \in I_{T}} \operatorname{MLO}(q)$ from model $\left(^{* *}\right)$, where $3 \nmid q \mid \frac{3^{\ell(q)}-1}{2}$

$I_{T}:=[(1-c) T, T]$ for $c=\frac{1}{2}$. More data points shown in Figure 3

The notation round $(x)$ stand for the closest integer to $x$, and the letters MLO stand for most likely outcome, since there is no other number more likely to occur as the value of $N_{q}$, under probabilistic model ${ }^{* *}$ ). 


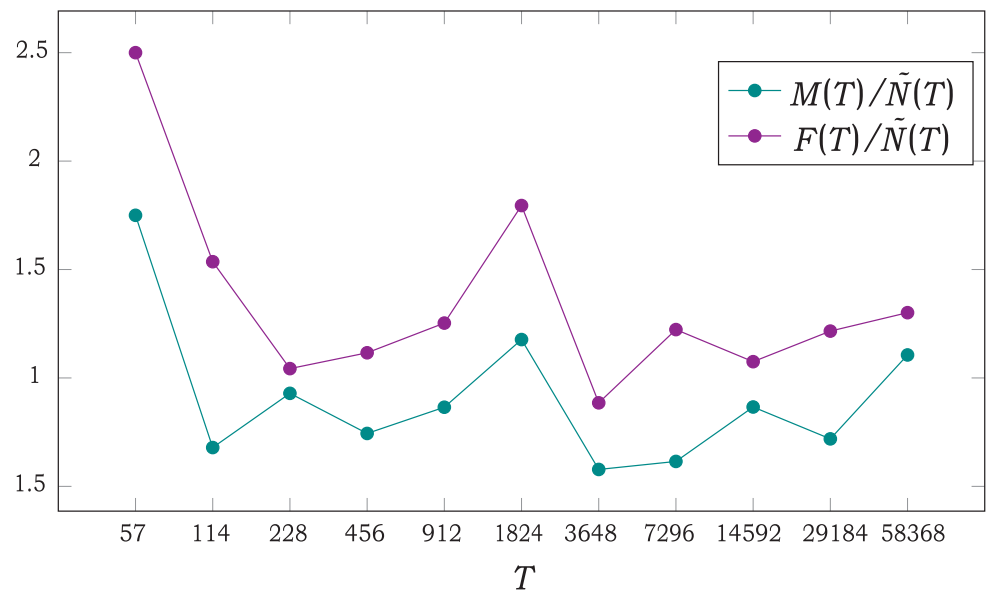

FIGURE 2. Ratios $\frac{M(T)}{\tilde{N}(T)}$ and $\frac{F(T)}{\tilde{N}(T)}$ for $c=\frac{1}{2}$. Our heuristic predicts that this graph tends to 1 at infinity.

Using inclusion/exclusion and Möbius inversion, one can show (for more details see [T]) that the number of even (as numbers in base 3) primitive words of length $\ell$ with symbols in the alphabet $\{0,1, \ldots, a-1\}$ is

$$
\sum_{d \mid \ell, \frac{\ell}{d} \text { even }} \mu\left(\frac{\ell}{d}\right) a^{d}+\sum_{d \mid \ell, \frac{\ell}{d} \text { odd }} \mu\left(\frac{\ell}{d}\right)\left\lceil\frac{a^{d}}{2}\right\rceil .
$$

As a consequence one obtains a simple formula for $\bar{m}(\ell, 3)$. This allows us to compute $\operatorname{MLO}(q)$ and hence to plot Figures 1 and 2. As can be seen in the Figures, within the range of our database of Cantor rationals, both models $\left(^{*}\right)$ and (**) give good approximations for the number of purely periodic Cantor rationals. The fit is not perfect though, and the plots reveal other interesting features. We try to explain some of these below.

\section{Remarks on fluctuations, Bourgain's theorem, and symmetries}

\subsection{Deviations from the mean}

An obvious objection to the line of reasoning presented above, is that our prediction for $\tilde{N}(T)$ is based on bounds on its expectation. That is, we have shown that our heuristic implies $\mathbb{E}(\widetilde{N}(T))=O\left(T^{d+\varepsilon}\right)$, but in order to justify $\widetilde{N}(T)=O\left(T^{d+\varepsilon}\right)$ one needs additional arguments, which we now briefly indicate. 
TABle 1. Denominators $q_{n}$ such that for all $q<q_{n+1}$ admitting Cantor rationals of denominator $q, \ell(q) / \log _{3} q \leq \ell\left(q_{n}\right) / \log _{3} q_{n}$. For all $q<3^{10}$ admitting Cantor rationals of denominator $q$, we have $\ell(q) / \log _{3} q \leq$ $\ell\left(q_{4}\right) / \log _{3} q_{4}$.

\begin{tabular}{|l||l|}
\hline$q_{n}$ & $\ell\left(q_{n}\right) / \log _{3} q_{n}$ \\
\hline \hline$q_{0}=3$ & 1.0 \\
$q_{1}=30$ & 1.292030029884618 \\
$q_{2}=84$ & 1.4876881693076203 \\
$q_{3}=146$ & 2.6453427135663814 \\
$q_{4}=386$ & 2.951356044207975 \\
\hline
\end{tabular}

TABle 2. The numbers $q=3^{r}+1, r=4, \ldots, 13$ where our heuristic gives poor predictions. When revising the prediction by a factor of $(3 / 2)^{r}$, which is the factor taking into account a symmetry $\omega \mapsto \omega \bar{\omega}$, we obtain a much better prediction.

\begin{tabular}{|r||c|c|c|c|c|}
\hline$r$ & $q=3^{r}+1$ & $N_{q}$ & $\mathrm{MLO}(q)$ & $\frac{N_{q}}{\mathrm{MLO}(q)}$ & $\left(\frac{2}{3}\right)^{r} \frac{N_{q}}{\operatorname{MLO}(q)}$ \\
\hline \hline 4 & 82 & 16 & 3 & 5.333 & 1.053 \\
5 & 244 & 30 & 4 & 7.5 & 0.988 \\
6 & 730 & 48 & 4 & 12 & 1.053 \\
7 & 2188 & 126 & 7 & 18 & 1.053 \\
8 & 6562 & 240 & 9 & 26.667 & 1.04 \\
9 & 19684 & 414 & 11 & 37.636 & 0.979 \\
10 & 59050 & 820 & 14 & 58.571 & 1.016 \\
11 & 177148 & 2024 & 23 & 88 & 1.017 \\
12 & 531442 & 4008 & 31 & 129.29 & 0.996 \\
13 & 1594324 & 8190 & 42 & 195 & 1.002 \\
\hline
\end{tabular}

If for some $\varepsilon>0$ there is an unbounded sequence of $T$ for which $\widetilde{N}(T) \geq$ $T^{d+\varepsilon}$, then (possibly modifying the constants $\varepsilon$ and $c$ ) we can take this to be a subsequence of the numbers in the form $T_{k}=(1+c)^{k}$. For each $k$ we let $X_{k}$ denote the random variable, in model $\left({ }^{*}\right)$, counting the number of $p / q \in \mathcal{C}$ with $q \in I_{T_{k}}$. We will show that the probability that $X_{k}$ exceeds $T_{k}^{d+\varepsilon}$ is $O\left(T_{k}^{-\varepsilon}\right)$, and hence is summable; from this it follows by Borel-Cantelli that the probability that for infinitely many $k$ we have $X_{k} \geq T_{k}^{d+\varepsilon}$ is zero. 


\section{ALEXANDER D. RAHM-NOAM SOLOMON-TARA TRAUTHWEIN—BARAK WEISS}

We continue to denote by $c^{\prime}, \lambda$ the constants as in $\S 4$, and write $T=T_{k}$ to simplify notation. Let $X_{k}^{(1)}$ (respectively, $X_{k}^{(2)}$ ) be the number of $p / q$ contributing to $X_{k}$ with $\ell(q)>\lambda \log _{3} T$ (respectively, $\log _{3} T+c^{\prime} \leq \ell(q) \leq \lambda \log _{3} q$ ). Let $\ell_{0}=\lambda \log _{3} T$, which is a lower bound for $\ell(q)$ when $p / q$ contributes to $X_{k}^{(1)}$. Since there are fewer than $T^{2}$ rationals $p / q$ with $q \in I_{T}$, the probability that $X_{k}^{(1)} \geq T^{d+\varepsilon}$ is smaller than the probability that a binomial random variable with probability

$$
p=\left(\frac{2}{3}\right)^{\ell_{0}}=T^{(d-1) \lambda}
$$

and $T^{2}$ trials we will have $T^{d+\varepsilon}$ successes. By the Markov inequality, this probability is bounded above by $T^{2+(d-1) \lambda-d-\varepsilon}=T^{-\varepsilon}$. The proof for $X_{k}^{(2)}$ is similar, again using the Markov inequality and the bounds used in the proof of (4.2).

\subsection{Large $\ell$ and Bourgain's theorem}

To highlight the sensitivity of $\tilde{N}(T)$ to fluctuations, consider the expression

$$
\widehat{\ell}(q)=\left\{\begin{array}{cc}
\ell(q), & N_{q} \neq 0 \\
0, & \text { otherwise }
\end{array} \quad\left(\text { with } N_{q} \text { as in (5.2) }\right)\right.
$$

that is, $\hat{\ell}(q)$ is the order of 3 in $(\mathbb{Z} / q \mathbb{Z})^{\times}$when there are rationals with denominator $q$ in $\mathcal{C}$, and zero otherwise. Clearly the nonzero values of $\widehat{\ell}(q)$ range between $\log _{3} q$ and $q$. If one could prove that $\widehat{\ell}(q) \ll \log _{3} q$ one would obtain a simple proof of Conjecture 1. Note that the heuristic behind Artin's conjecture (see $\mathrm{Mo}$ ) predicts that there are infinitely many $q$ for which $\ell(q) \gg q$, so that this may appear at first sight to be wildly optimistic. However our restriction $N_{q} \neq 0$ is a stringent one. In fact, our computations found that for all $3 \leq q<3^{10}, \widehat{\ell}(q)<3 \log _{3} q$ (see Table 1). This gives some evidence toward the following conjecture of Fishman and Simmons [FS, Conj. 5.6]: for some $c>0$, there are only finitely many Cantor rational $q$ for which $\hat{\ell}(q) \geq c \log q$.

On the other hand, by observation (ii), a large value of $\widehat{\ell}(q)$ would make a large contribution to $\tilde{N}(T)$ when $q \in I_{T}$. For example, if there were infinitely many $q$ for which $\widehat{\ell}(q)>q^{d+\varepsilon}$, then their contribution alone would yield a contradiction to Conjecture 1, However, a difficult result of Bourgain [B] implies that for any $\delta>0, \widehat{\ell}(q) \ll q^{\delta}$. Bourgain's theorem is much stronger inasmuch as it implies that the cosets of the subgroup $H$ equidistribute in the interval $[0,1]$ when $\ell(q)>q^{\delta}$, while to obtain the upper bound above, one only needs to know that if $\ell(q)>q^{\delta}$, then any coset for $H$ contains at least one point in the interval $(1 / 3,2 / 3)$. It would be of interest to obtain better upper bounds on $\widehat{\ell}(q)$ than those implied by Bourgain's theorem. 


\section{RATIONALS IN THE CANTOR SET}

\subsection{Additional sources of fluctuations}

It is easy to show that (4.4) predicts a lower bound $\widetilde{N}(T) \gg T^{d}$. However we do not expect a precise asymptotic in the form $\widetilde{N}(T) \sim c T^{d}$, that is, we do not expect the limit of $\widetilde{N}(T) / T^{d}$ to exist. There are two reasons for fluctuations in this expression. First consider the numbers of the form $q=\left(3^{\ell}-1\right) / 2$, for which $\ell(q)=\ell$. If $c<2 / 3$, depending on the choice of $T$, the range $I_{T}$ may or may not contain one such number. In case it does, this contributes a term of order $(2 / 3)^{\ell} \asymp T^{d}$ to the sum, which would contribute to the main term. Thus we have fluctuations according as the window $I_{T}$ does or does not contain such $q$, or for general $c \in(0,1)$, depending on the number of such $q$ in the interval $I_{T}$. See Figure 3 ,

Although these fluctuations would contradict a precise asymptotic $\widetilde{N}(T) \sim c T^{d}$, they do not preclude the weaker statement $\widetilde{N}(T) \asymp T^{d}$. A potentially more seri-

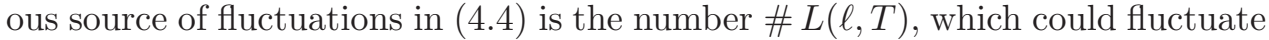
considerably due to fluctuations in the numbers $\tau\left(3^{\ell}-1\right)$. It would be interesting to determine the asymptotic behavior of the right hand side of (4.4).

\subsection{Symmetries}

Heuristics $\left(^{*}\right)$ and $\left({ }^{* *}\right)$ can also be used to make predictions for the number $N_{q}$ of Cantor rationals with a fixed denominator $q$. However in this regime, our computations reveal many values of $q$ for which the heuristic gives inaccurate predictions. Some of these are shown in Tables 2 and 3 . The numbers in Table 2 are all of the form $3^{r}+1$, and in Table 3 we show all numbers $q$ for which $\ell(q)=24$ and the prediction is inaccurate by a factor of 4 or more. We will consider a possible explanation for these inaccuracies by introducing a (non-rigorous) notion of 'symmetries' in base 3 expansion.

The identity $\frac{3^{2 r}-1}{2}=\frac{\left(3^{r}-1\right)\left(3^{r}+1\right)}{2}$ easily implies the following (we leave details to the reader): suppose a purely periodic rational in base 3 expansion has repeating block $\omega \in\{0,2\}^{r}$, where $r$ is the length of $\omega$, and $\bar{\omega}$ is the block obtained from $\omega$ by replacing occurences of 0 with 2 and 2 with 0 . Then the word $\omega \bar{\omega}$ of length $2 r$ obtained by concatenating $\omega, \bar{\omega}$ defines (via an infinite base 3 expansion $0 . \omega \bar{\omega} \omega \bar{\omega} \ldots)$ a number in $\mathcal{C}$ whose denominator divides $3^{r}+1$. This implies

that any $\frac{p}{3^{r}-1} \in \mathcal{C}$ gives rise to some $\frac{p^{\prime}}{3^{r}+1} \in \mathcal{C}$ (and in fact, by observation (ii) in $\S$, to the $\times 3$-orbit of this word, which typically contains $2 r$ numbers). It can be deduced that heuristic ( $\left.{ }^{* *}\right)$ underestimates numbers $p^{\prime} / q^{\prime}$ with $q^{\prime}$ dividing $3^{r+1}$, arising in this way, by a factor of approximately $(3 / 2)^{r}$. The revised heuristic is borne out by Table 2, where the last column corrects heuristic $\left({ }^{* *}\right)$ by this factor, giving a good fit with the data. 
ALEXANDER D. RAHM-NOAM SOLOMON-TARA TRAUTHWEIN-BARAK WEISS

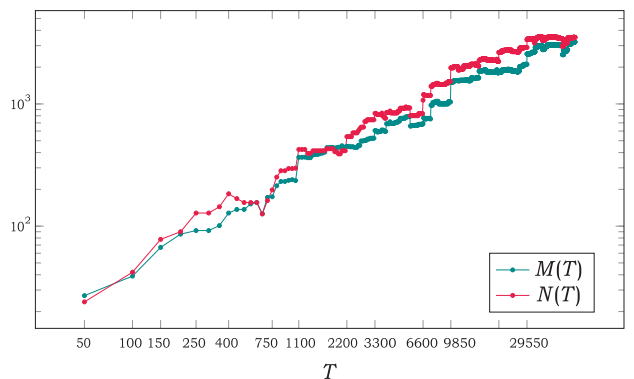

(A) $c=0.25$

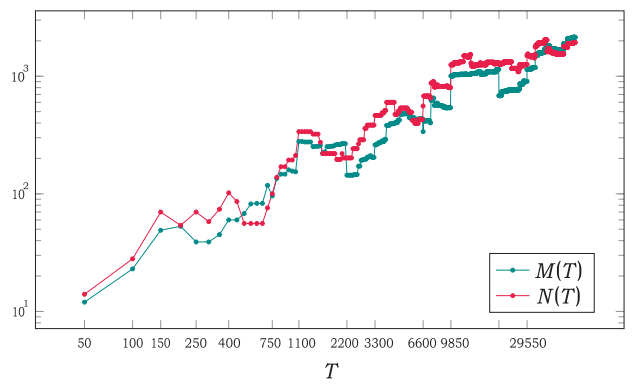

(C) $c=0.5$

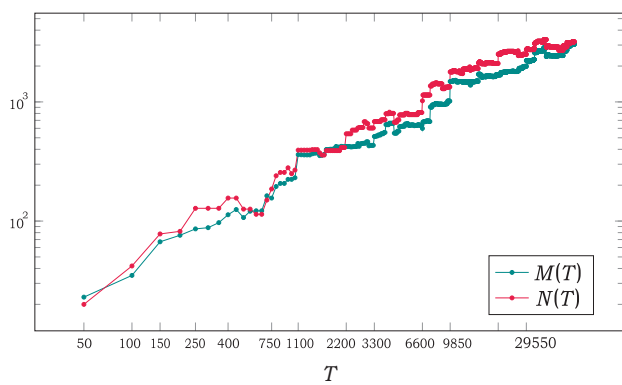

(в) $c=0$

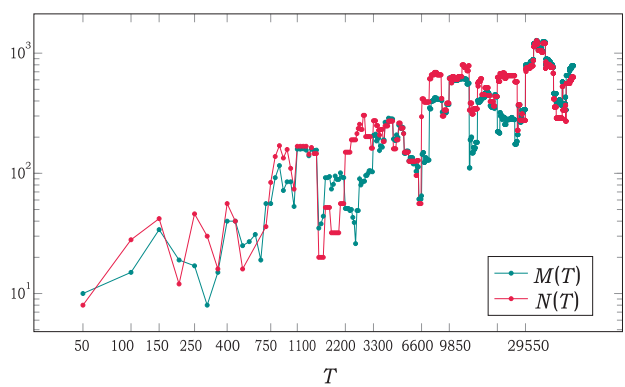

(D) $c=0.25$

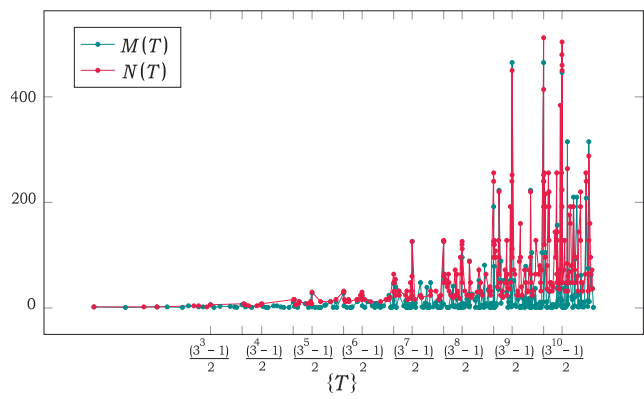

(E) $c=0$

Figure 3. For different values of $c$ (which determine the intervals $I_{T}:=$ $[(1-c) T, T])$, we plot the summed number of purely periodic Cantor rationals $\tilde{N}(T)$ and its approximation $M(T)$ from model $\left({ }^{* *}\right)$. As predicted in $\S 6.3$ there are more fluctuations for smaller $c$.

The mapping $\omega \mapsto \omega \bar{\omega}$ used above is for us an example of a symmetry in base 3. Here is another example. Suppose $\omega, \bar{\omega} \in\{0,2\}^{r}$ are as in the previous paragraph, and suppose $\mathbf{0}$ and $\mathbf{2}$ denote strings of length $r$ consisting only of the digit 0 (respectively 2 ). Then one may check, this time using the identity $3^{3 r}-1=\left(3^{r}-1\right)\left(3^{2 r}+3^{r}+1\right)$, that repeating blocks $\omega \bar{\omega} \mathbf{0}$ and $\omega \bar{\omega} \mathbf{2}$ give numbers 
in $\mathcal{C}$ whose denominator divides $3^{2 r}+3^{r}+1$. For example, taking $r=7$, we have $q=3^{14}+3^{7}+1=4785157$, our heuristic $\left.{ }^{* *}\right)$ gives $\operatorname{MLO}(q)=1771$, and our computer program finds $N_{q}=4158$, which is a poor fit. The number of strings of the form $\omega \bar{\omega} \mathbf{0}$ and $\omega \bar{\omega} \mathbf{2}$, along with all their cyclic permutations (taking into account observation (ii) in $\$ 5$ ) is 2562. Some of these give a subset of the ones already considered in heuristic $\left(^{* *}\right)$, so taking this symmetry into account we should expect $2562 \cdot \frac{\phi(q)}{q}=2365 \leq N_{q}$. This indeed gives a better (albeit still not very precise) prediction. We suspect that there are more symmetries contributing to the numbers $N_{q}$ and hope to return to this issue in future work. In Table 5 we have tabulated the numbers $q_{r}$ for $r=2, \ldots, 10$, along with the numbers of strings of the above form multiplied by $\phi(q) / q$, and compared this prediction with the actual number of strings of this form which are reduced rationals with denominator $q_{r}$.

When $\ell=k r$ for $k, r \in \mathbb{N}, k \geq 2$, we can often make a similar construction of a repeating block of length $\ell$ which is composed of $k$ sub-blocks of size $r$ (in the preceding two paragraphs we gave examples with $k=2,3$ ). The result will be that for the numbers

$$
q=3^{(k-1) r}+3^{(k-2) r}+\cdots+3^{r}+1,
$$

$N_{q}$ will be significantly larger than predicted by our heuristic. The same will be true for large divisors $q^{\prime}$ of such $q$. Thus if $\ell$ has many divisors, there will be many values of $q$ for which our predictions will be poor. In all of them we expect our heuristic to give a number which is smaller than the correct value, and we do not expect such very poor predictions to occur when $\ell$ is prime. These two expectations are borne out in Tables 3 and 4 below. We invite the reader to try to find explanations for the numbers appearing in Table 3 note that we have explained the appearance of 531442 using a symmetry $\omega \mapsto \omega \bar{\omega}$, and that 589771 and 84253 are large divisors of $3^{16}+3^{8}+1$ and can thus be explained using the symmetries $\omega \mapsto \omega \bar{\omega} \mathbf{0}, \omega \mapsto \omega \bar{\omega} \mathbf{2}$.

\section{Appendix A. Computing the Cantor rationals of given denominator}

In this appendix, we give an algorithm to compute the set of rational numbers in the Cantor set of given denominator $q$, namely the Cantor rationals of reduced form $\frac{p}{q}$. It is stated in Algorithm 1 below, and has been implemented by the authors in Pari/GP. We denote by $\ell(q)$ the order of the element 3 in the group of multiplicative units in the ring $\mathbb{Z} / q \mathbb{Z}$ with $q$ elements. 


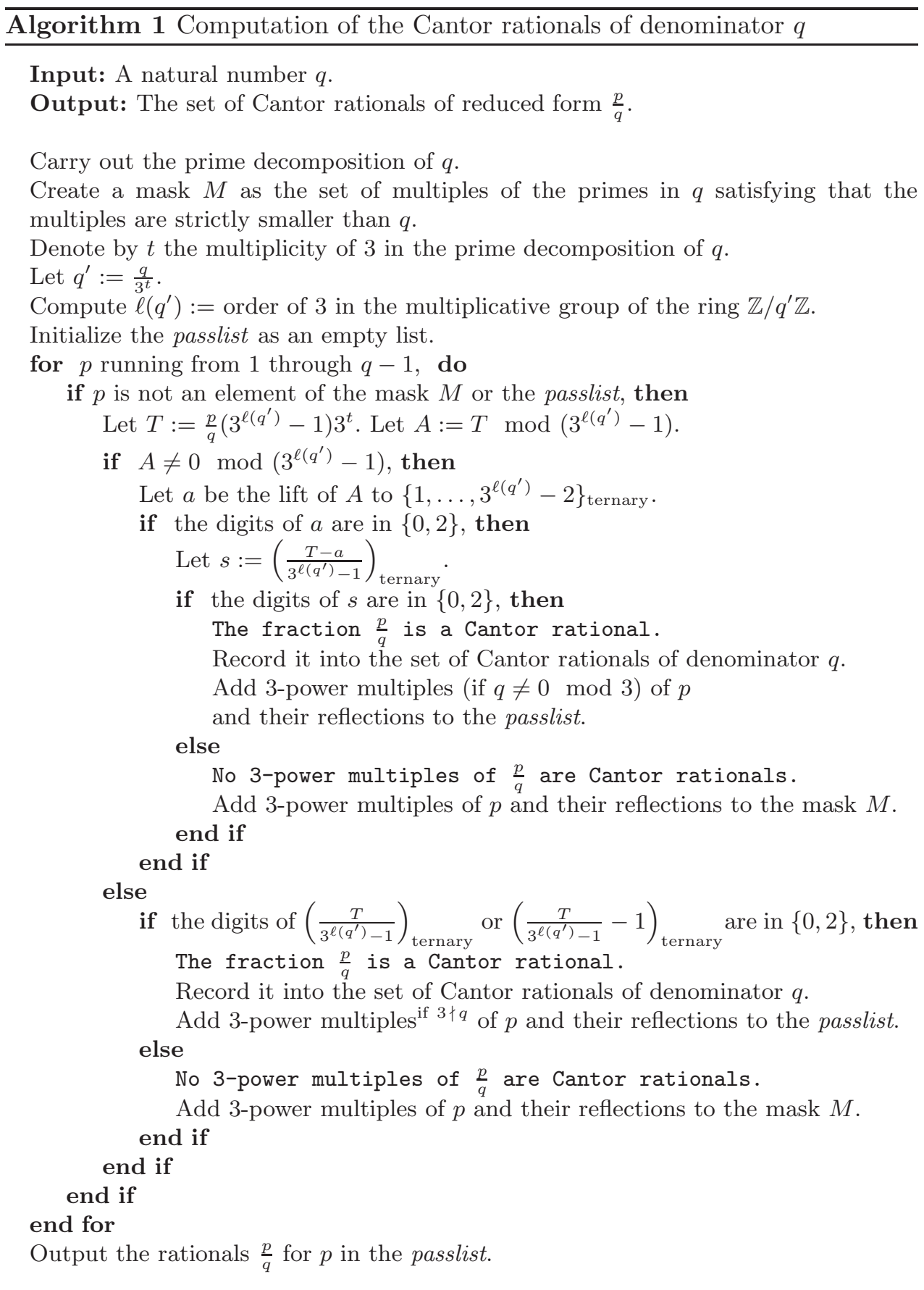


TABLE 3. All values of $q$ with $\ell(q)=24$ for which our heuristic makes a prediction which is incorrect by a factor of 4 or more. Note that in all of these examples, $N_{q}>\operatorname{MLO}(q)$. At least three, and probably all, of the entries in the table are related to the symmetries discussed in $\S$ refsubsec: symmetries.

\begin{tabular}{|c||c|c|c|c|}
\hline$q$ & $\ell(q)$ & $N_{q}$ & $\mathrm{MLO}(q)$ & $\frac{N_{q}}{\mathrm{MLO}(q)}$ \\
\hline \hline 12962 & 24 & 72 & 1 & 72 \\
14965 & 24 & 48 & 1 & 48 \\
29848 & 24 & 48 & 1 & 48 \\
84253 & 24 & 96 & 9 & 10.391 \\
129620 & 24 & 48 & 6 & 8 \\
181468 & 24 & 96 & 9 & 10.391 \\
239440 & 24 & 96 & 11 & 8.727 \\
259240 & 24 & 48 & 12 & 4 \\
298480 & 24 & 96 & 11 & 8.727 \\
531442 & 24 & 4008 & 31 & 129.29 \\
589771 & 24 & 336 & 55 & 6.109 \\
4731130 & 24 & 960 & 222 & 4.324 \\
21257680 & 24 & 4176 & 985 & 4.24 \\
\hline
\end{tabular}

Proposition A.1. The set computed by Algorithm 1 contains all the Cantor rationals of denominator $q$ for its reduced form. This algorithm terminates within finite time.

Proof.

- The period length of $\frac{p}{q}$ in the ternary system is given by $\ell\left(q^{\prime}\right)$. Hence, the finite sequence $a$ of ternary digits is precisely the periodical sequence in $\frac{p}{q}$. Furthermore,

$$
\frac{s\left(3^{\ell\left(q^{\prime}\right)}-1\right)+a}{\left(3^{\ell\left(q^{\prime}\right)}-1\right) 3^{t}}=\frac{p}{q} .
$$

So, the sequence $s$ is precisely the sequence of ternary digits preceding the periodical part in the ternary expansion of $\frac{p}{q}$. By the elementary ternary digits property of the Cantor set, Algorithm 1 decides if $\frac{p}{q}$ is a Cantor rational. The mask $M$ allows it to check all suitable fractions $\frac{p}{q}$. Here, and for establishing the passlist, we make use of the well-known symmetry of the Cantor set: If $x$ is an element of the Cantor set, then the same holds for $(1-x), \frac{x}{3}$, and - provided that it is in the unit interval - $3 x$. 


\section{ALEXANDER D. RAHM-NOAM SOLOMON-TARA TRAUTHWEIN—BARAK WEISS}

- The loop in Algorithm 1 consists of $(q-1)$ repetitions, which contain a finite number of finite-time steps.

\section{REMARK A.2.}

- The mask $M$ can be omitted and a coprimality check for $(p, q)$ inserted, to obtain a simpler algorithm which is mathematically equivalent to Algorithm 1. The difference lies in the efficiency: In fact, the mask $M$ is a powerful tool to reduce the time needed to carry out the algorithm, minimizing the number of iterations of most expensive steps, which grows fast with $q$.

- Even more important for the efficiency is the sub-algorithm testing the belonging of the ternary digits to the set $\{0,2\}$, because the numbers to be tested are incredibly great integers.

TABLE 4. Some numbers $q<\frac{3^{\ell(q)}-1}{2}$ for which $\ell(q)$ is a prime, including all such $q$ with $11 \leq \ell(q) \leq 23$. In this case, symmetries are impossible and our heuristic works well for each individual $q$.

\begin{tabular}{|c||c|c|c|c|}
\hline$q$ & $\ell(q)$ & $N_{q}$ & $\operatorname{MLO}(q)$ & $\frac{N_{q}}{\operatorname{MLO}(q)}$ \\
\hline \hline 23 & 11 & 0 & 0 & - \\
47 & 23 & 0 & 0 & - \\
683 & 31 & 0 & 0 & - \\
1597 & 19 & 0 & 1 & 0 \\
1871 & 17 & 0 & 4 & 0 \\
3851 & 11 & 88 & 89 & 0.989 \\
28537 & 29 & 0 & 0 & - \\
34511 & 17 & 68 & 70 & 0.971 \\
102673 & 31 & 0 & 1 & 0 \\
363889 & 19 & 304 & 328 & 0.927 \\
$59 \cdot 28537$ & 29 & 0 & 26 & 0 \\
4404047 & 31 & 62 & 31 & 2 \\
20381027 & 29 & 232 & 319 & 0.727 \\
1001523179 & 23 & 178480 & 178481 & 0.999994 \\
\hline
\end{tabular}




\section{RATIONALS IN THE CANTOR SET}

TABLE 5. The numbers $q_{r}=3^{2 r}+3^{r}+1$ with the contribution of the symmetries of the form $\omega \mapsto \omega \bar{\omega} \mathbf{0}$ and $\omega \mapsto \omega \bar{\omega} \mathbf{2}$. The number $X_{r}$ counts all strings of length $3 r$ of the specified form, $Y_{r}=\left\lfloor X_{r} \cdot \frac{\phi\left(q_{r}\right)}{q_{r}}\right\rfloor$, and $Z_{r}$ is the actual number of Cantor rationals with denominator $q_{r}$ of this special form.

\begin{tabular}{|r||c|c|c|c|c|c|}
\hline$r$ & $q_{r}$ & $N_{q_{r}}$ & $X_{r}$ & $Y_{r}$ & $Z_{r}$ & $Y_{r}+\operatorname{MLO}\left(q_{r}\right)$ \\
\hline \hline 1 & 13 & 6 & 6 & 6 & 6 & 13 \\
2 & 91 & 12 & 18 & 14 & 12 & 27 \\
3 & 757 & 54 & 54 & 54 & 54 & 93 \\
4 & 6643 & 120 & 156 & 122 & 120 & 202 \\
5 & 59293 & 450 & 420 & 388 & 390 & 638 \\
6 & 532171 & 1368 & 1062 & 978 & 1008 & 1641 \\
7 & 4785157 & 4158 & 2562 & 2365 & 2436 & 4136 \\
8 & 43053283 & 9744 & 5976 & 4663 & 4560 & 8654 \\
9 & 387440173 & 38988 & 13608 & 13450 & 13500 & 26931 \\
10 & 3486843451 & 91440 & 30450 & 23224 & 23520 & 50961 \\
\hline
\end{tabular}

ACKnowledgements. We thank Johannes Schleischitz and David Simmons for comments on an earlier version of this paper.

\section{REFERENCES}

[B] BOURGAIN, J.: Exponential sum estimates over subgroups of $Z_{q}^{\times}$, q arbitrary, J. Anal. Math. 97 (2005), 317-356.

[BFR] BRODERICK, R.-FISHMAN, L.-REICH, A.: Intrinsic Approximation on Cantorlike Sets, a Problem of Mahler, Mosc. J. Comb. Number Theory 1 (2011), 3-12.

[Bu] BUGEAUD, Y.: Diophantine approximation and Cantor sets, Math. Ann. 341 (2008), no. 3, 677-684.

[E] ERDŐS, P.: On the sum $\sum_{d \mid 2^{n}-1} d^{-1}$, Israel J. Math. 9 (1971), 43-48.

[F] FISHMAN, L.: Schmidt's game on fractals, Israel J. Math. 171 (2009), 77-92.

[FS] FISHMAN, L.-SIMMONS, D.: Intrinsic approximation for fractals defined by rational iterated function systems: Mahler's research suggestion, Proc. Lond. Math. Soc.(3) 109 (2014), no. 1, 189-212.

[LSV] LEVESLEY, J.-SALP, C.-VELANI, S.: On a problem of K. Mahler: Diophantine approximation and Cantor sets, Math. Ann. 338 (2007), no. 1, 97-118.

[M] MAHLER, K.: Some suggestions for further research, Bull. Aust. Math. Soc. 29 (1984), no. 1, 101-108.

[Mo] MOREE, P.: Artin's primitive root conjecture - a survey, Integers 12 (2012), no. 6, $1305-1416$. 


\section{ALEXANDER D. RAHM-NOAM SOLOMON-TARA TRAUTHWEIN-BARAK WEISS}

[Sch] SCHLEISCHITZ, J.: On intrinsic and extrinsic rational approximation to Cantor sets, Ergodic Theory and Dynamical Systems (2020), 1-30. (DOI:10.1017/etds.2020.7).

[S] SHPARLINSKI, I.: On the arithmetic structure of rational numbers in the Cantor set, Bull. Aust. Math. Soc. (2020), 1-6 (doi:10.1017/S0004972720000386).

[SW] SIMMONS, D.-WEISS, B.: Random walks on homogeneous spaces and Diophantine approximation on fractals, Invent. Math. 216 (2019), 337-394.

[T] TRAUTHWEIN, T.: Approximation of Cantor Rational Cardinalities by Primitive Words, Master 1 project report (2019), Experimental Mathematics Lab, University of Luxembourg,

http://math.uni.lu/eml/projects/reports/Cantor_rationals_project_report.pdf

[W] WEISS, B.: Almost no points on a Cantor set are very well approximable, R. Soc. Lond. Proc. Ser. A Math. Phys. Eng. Sci. 457 (2001), 949-952.

Received May 23, 2020

Accepted September 27, 2020
Alexander D. Rahm

University of French Polynesia

GAATI Laboratory

BP 6570, 98702 Faa'a

FRENCH POLYNESIA

E-mail: Alexander.Rahm@upf.pf

\section{Noam Solomon}

Massachusetts Institute of Technology

77 Massachusetts Avenue

Cambridge, MA 02139

U.S.A

E-mail: noams@mit.edu

\section{Tara Trauthwein}

University of Luxembourg

Department of Mathematics

2 av. de l'Université

L-4365 Esch-sur-Alzette

LUXEMBOURG

E-mail: tara.trauthwein@uni.lu

\section{Barak Weiss}

Tel Aviv University

Department of Mathematics

Tel-Aviv, 69978

ISRAEL

E-mail: barakw@tauex.tau.ac.il 